\title{
Interaction of a magnetized pulsar wind with its surroundings
}

\section{MHD simulations of pulsar wind nebulae}

\author{
E. van der Swaluw ${ }^{\star}$ \\ Dublin Institute for Advanced Studies, 5 Merrion Square, Dublin 2, Ireland \\ Received 16 September 2002 / Accepted 18 March 2003

\begin{abstract}
Magnetohydrodynamical simulations are presented of a magnetized pulsar wind interacting directly with the interstellar medium, or, in the case of a surrounding supernova remnant, with the associated freely expanding ejecta of the progenitor star. In both cases the simulations show that the pulsar wind nebula will be elongated due to the dynamical influence of the toroidal magnetic fields, which confirm predictions from a semi-analytical model presented by Begelman \& Li. The simulations follow the expansion of the pulsar wind nebula when the latter is bounded by a strong shock and show that the expansion can be modeled with a standard power-law expansion rate. By performing different simulations with different magnetization parameters, I show that the latter weakly correlates with the elongation of the pulsar wind nebula. The results from the simulations are applied to determine the nature of the expansion rate of the pulsar wind nebula 3C 58. It is shown that there is both observational and theoretical evidence which supports the scenario in which the pulsar wind nebula 3C 58 has caught up with the reverse shock of the associated (but undetected) supernova remnant.
\end{abstract}

Key words. pulsars: general - supernova remnants - shock waves - hydrodynamics

\section{Introduction}

After the explosion of a massive star as a supernova, the progenitor star collapsed into a compact object, which can be a neutron star or a black hole. If the neutron star manifests itself as a rapidly rotating pulsar, the spin-down energy of the pulsar is converted into a relativistic pulsar wind (Rees \& Gunn 1974), which blows a bubble or pulsar wind nebula (PWN) into the surrounding medium. The observational counterpart of a PWN is a plerion, which is characterized by filled-center diffuse emission (Weiler \& Panagia 1978). For young plerionic supernova remnants, the central region contains the active young pulsar.

The supernova explosion itself drives a blastwave into the interstellar medium (ISM), which results in an expanding shell of hot (shocked) material. The observational counterpart is a shell type supernova remnant, which is characterized by extended emission from the roughly spherical shell bounding the supernova remnant (SNR). Due to the deceleration of the expanding SNR by the surrounding ISM, a reverse shock forms (McKee 1974), which will propagate into the interior of the SNR when the amount of swept-up material becomes comparable with the ejected mass of the progenitor star. In the case of a SNR which contains both, an expanding shell of hot shocked material and a centered PWN (which is the observational counterpart of a composite remnant), the interaction between both components becomes significant when the reverse shock hits

\footnotetext{
* e-mail: swaluw@rijnh.nl
}

the PWN (Reynolds \& Chevalier 1984; van der Swaluw et al. 2001; Blondin et al. 2001). After the passage of the reverse shock the PWN will make a transition from a supersonic to a subsonic expansion (van der Swaluw et al. 2001). The PWN will be deformed into a bow shock nebula when the motion of the pulsar becomes supersonic as the pulsar approaches the shell of the SNR (van der Swaluw et al. 2003).

In this Paper I will focus on young PWNe which are expanding supersonically, where the surrounding medium can be either the ISM or the freely expanding ejecta of the progenitor star (i.e. the stage of the PWN before it encounters the reverse shock). For both scenarios there exists observational evidence. In the case of SNR G11.2-0.3 a large PWN radius with respect to the shell of the SNR is detected at radio frequencies (Tam et al. 2002), making the system a probable candidate for a PWN which has not encountered the reverse shock yet. In the case of the Crab Nebula there is indirect evidence for the presence of a shell containing shocked material and fast moving ejecta around the pulsar wind nebula (Sankrit \& Hester 1997). There are some counter-examples, naked plerions like G74.9+1.2 and G63.7+1.1, where there is no evidence for any shell or fast-moving ejecta around the plerionic component (see e.g. Wallace et al. 1997a,b). This suggests that the pulsar wind in these systems is interacting directly with the surrounding ISM.

A common feature of most plerions is a morphology with a significant elongation. This elongation is most strongly apparent at radio frequencies, where the complete bubble can be 
observed because of the long lifetime of radio electrons with respect to the age of the PWN itself. Begelman \& Li (1992) (hereafter B\&L) explain the elongation of the Crab Nebula by considering an axially symmetric model, where the elongation results from the dynamical influence of the toroidal magnetic fields in the pulsar wind bubble. Their model is a steadystate model, in contrast with recent time-dependent models of PWNe evolving inside SNRs as discussed by van der Swaluw et al. (2001) and Blondin et al. (2001). However, these authors performed hydrodynamical simulations, and thus could not consider the dynamical influence of the magnetic fields on the PWN evolution.

In this Paper I consider the time-dependent evolution of a supersonically expanding PWN, driven by a magnetized pulsar wind. The evolution of the PWN is modeled by performing magnetohydrodynamical (MHD) simulations using the Versatile Advection Code $^{1}$ (VAC). I confirm the earlier analytical results from B\&L, which state that the elongated morphology of the Crab Nebula (and other plerions) can be explained by the dynamical influence of the toroidal magnetic fields in the pulsar wind bubble.

I trace the position of the shock during the evolution of the PWN and show that although the magnetic fields are dynamically important, the expansion rate can still be modeled by a power-law expansion. I show that during the overall expansion the elongation will increase at a very slow rate, and it is at the very early stage of the PWN that its shape will be significantly elongated. Furthermore I will discuss the importance of the effects of the magnetization parameter $\sigma$, i.e. the standard ratio of the Poynting flux to the plasma kinetic energy flux, in the pulsar wind on the evolution of the PWN. Finally I will discuss the implications of the current simulations on the interpretation of the evolutionary status of the PWN 3C 58.

\section{Evolution of a pulsar wind bubble}

\subsection{The hydrodynamical limit}

I consider the evolution of a supersonically expanding PWN. This type of expansion occurs when the pulsar wind is directly interacting with the ISM or with the freely expanding ejecta of a young SNR. In this section I consider the hydrodynamical limit, i.e. no magnetic fields are involved, which enables one to use simple power-law expansion rates for the radius of the $\operatorname{PWN}\left(R_{\mathrm{pwn}} \propto t^{\alpha}\right)$.

In the case of a pulsar wind with a constant luminosity $L_{0}$, interacting with the ISM with a uniform density $\rho_{0}$, the eqation for the radius of the PWN is similar to the equation for stellar wind bubbles (Castor et al. 1975), i.e.

$R_{\mathrm{pwn}}=\bar{C}\left(\frac{L_{0}}{\rho_{0}}\right)^{1 / 5} t^{3 / 5}$,

where $\bar{C}$ is of the order of unity. In the case of a PWN expanding in the freely expanding ejecta of a SNR, the equation for

\footnotetext{
${ }^{1}$ See http://www.phys.uu.nl/ ${ }^{\sim}$ toth/
}

the radius of the PWN was derived by van der Swaluw et al. (2001):

$R_{\mathrm{pwn}}=C\left(\frac{L_{0} t}{E_{0}}\right)^{1 / 5} V_{0} t \propto t^{6 / 5}$,

here $L_{0}$ is the spindown luminosity, $E_{0}$ is the explosion energy of the SNR, $C$ is of the order of unity and $V_{0}$ is defined by:

$E_{0}=\frac{3}{10} M_{\mathrm{ej}} V_{0}^{2}$

here $M_{\mathrm{ej}}$ denotes the mass of the ejecta.

The ratio $\mathcal{R}$ between the radius of a PWN expanding into the ISM (Eq. (1)) with respect to a PWN expanding into freely expanding ejecta (Eq. (2)) is of the order of unity for supersonically expanding PWNe (using $\bar{C}=C=1$ ):

$\mathcal{R} \simeq 2.36\left(\frac{n_{0}}{E_{51}}\right)^{1 / 5} V_{10000} t_{\mathrm{kyr}}^{3 / 5}$,

here $V_{10000}$ is expressed in units of $10000 \mathrm{~km} \mathrm{~s}^{-1}, E_{51}$ is the eplosion energy in units of $10^{51} \mathrm{ergs}, n_{0}$ is the hydrogen number density of the ISM in units of $\mathrm{cm}^{-3}$ and $t_{\mathrm{kyr}}$ is the age expressed in units of 1000 years. The simulations indeed show an enhanced expansion rate for the pulsar wind expanding into the ISM for the considered ages $\left(t_{\mathrm{kyr}} \simeq 1\right)$. This can be explained by the fact that the pulsar wind embedded in the freely expanding ejecta will start to blow its bubble into the very dense medium of the ejected material from the supernova explosion. However, as the ejecta is freely expanding the density will decrease as a function of time $\left(\rho_{\mathrm{ej}} \propto t^{-3}\right)$ which will accelerate the overall expansion of the pulsar wind bubble (e.g. Chevalier 1982; van der Swaluw et al. 2001). This behaviour is reflected in the equations above by inserting $\rho_{0} \sim t^{-3}$ into Eq. (1) which yields the power-law behaviour of Eq. (2): the expansion of the pulsar wind bubble starts at a slow rate due to the dense medium but accelerates to high expansion rates as the density of the ejecta is uniformly decreasing.

I will use the MHD simulations to investigate the expansion rate of a magnetized $\mathrm{PWN}$, and compare the expansion rate with the above power-law expansion rates.

\subsection{A magnetized quasi-static pulsar wind nebula}

A magnetized pulsar wind is driven by the spin-down luminosity of a rapidly rotating pulsar. The pulsar wind has a high Lorentz factor (in the case of the Crab $\Gamma_{\mathrm{w}} \geq 10^{3}$ ) (Gallant et al. 2002) and is terminated by a strong MHD shock, where the wind pressure and the confining ram pressure balance. At the position of the wind termination shock $R_{\mathrm{ts}}$ one can write the luminosity of the pulsar wind as a combination of particle energy and magnetic energy (Kennel \& Coroniti 1984):

$L=4 \pi \Gamma_{\mathrm{w}}^{2} n_{\mathrm{w}} R_{\mathrm{ts}}^{2} m c^{3}(1+\sigma)$,

where $n_{\mathrm{w}}$ is the proper density in the wind, $m$ the mean mass per particle and $\sigma$ is the ratio of magnetic energy to particle energy, i.e.:

$\sigma=\frac{B^{2}}{4 \pi n_{\mathrm{w}} \Gamma_{\mathrm{w}} m c^{2}}$, 
where $B$ is the magnetic field component, which I will assume to be purely toroidal:

$\boldsymbol{B}=B(r, z) \hat{\boldsymbol{\phi}}$,

using an axially symmetric configuration $(r, \phi, z)$.

The shock heats the incoming flow and yields a high sound speed, $c_{\mathrm{S}} \sim c / \sqrt{3}$ downstream of the MHD shock (Kennel \& Coroniti 1984).

$\mathrm{B} \& \mathrm{~L}$ described the interior of a PWN by considering a steady-state solution $(\partial / \partial t=0)$ for the ideal MHD equations in an axially symmetric configuration. Due to the high sound speed in the PWN, pressure imbalances are quickly smoothed out, therefore one can write the momentum equation as:

$\boldsymbol{\nabla}\left(P+\frac{B^{2}}{8 \pi}\right)+\frac{B^{2}}{4 \pi r} \hat{\boldsymbol{r}}=0$,

i.e.

$\frac{\partial}{\partial z}\left(P+\frac{B^{2}}{8 \pi}\right)=0$

and

$\frac{\partial}{\partial r}\left(P+\frac{B^{2}}{8 \pi}\right)=-\frac{B^{2}}{4 \pi r}$.

From these two equations one can immediately observe that the total pressure along the symmetry axis (the $z$-axis) will be constant (Eq. (8)), whereas the total pressure along the radial axis will decrease; the pressure gradient in the radial direction balances the magnetic pinching force (the RHS of Eq. (9)), therefore the total pressure long the $r$-axis will decrease (Eq. (9)). B\&L show that the resulting difference along the PWN shock in pressure between the interior and the exterior of the bubble will result in an elongation of the PWN, as is observed in the Crab Nebula today.

\section{MHD simulations}

\subsection{MHD calculations of a pulsar wind}

The difference between the model presented in this paper and the model of B\&L is that I integrate the complete set of MHD equations in the non-relativistic limit with a continuous (thermal and magnetic) energy and mass input in a region centered around the position of the pulsar to model the pulsar wind. This yields the complete evolution of a PWN, for which one can trace the expansion, elongation rate and the internal structure, without being restricted to a quasi-static model as in B\&L.

I use the Versatile Advection Code (VAC; Tóth 1996) to integrate the equations of ideal MHD:

$$
\begin{aligned}
& \frac{\partial \rho}{\partial t}+\nabla \cdot(\rho \boldsymbol{V})=0, \\
& \frac{\partial(\rho \boldsymbol{V})}{\partial t}+\nabla \cdot\left(\rho \boldsymbol{V} \otimes \boldsymbol{V}+P \boldsymbol{I}-\frac{\boldsymbol{B} \otimes \boldsymbol{B}}{8 \pi}\right)=0, \\
& \frac{\partial}{\partial t}\left(\frac{1}{2} \rho V^{2}+\frac{B^{2}}{8 \pi}+\rho e\right)+\boldsymbol{\nabla} \cdot\left(\rho \boldsymbol{V}\left(\frac{1}{2} V^{2}+h-\frac{B^{2}}{8 \pi}\right)\right)=0,
\end{aligned}
$$

$\frac{\partial \boldsymbol{B}}{\partial t}+\boldsymbol{\nabla} \times(\boldsymbol{V} \times \boldsymbol{B})=0$,

here $h$ is the enthalpy,

$h \equiv e+\frac{P}{\rho}$,

$\rho$ is the mass density, $\boldsymbol{V}$ is the velocity, $B$ is the magnetic field strength, $e$ is the internal energy density and $P$ is the thermal pressure.

I solve the MHD equations on a uniform grid in an axially symmetric configuration $(r, \phi, z)$ in $2.5 \mathrm{D}$. In a $2.5 \mathrm{D}$ simulation all the flow variables $\mathcal{F}_{i}$ (where $i=r, \phi, z$ ) are solved as a function of $r$ and $z$, which includes the dynamical influence of the flow variables in the $\phi$ direction, but the flow variables in this direction are constant at a given $r$ and $z$, i.e. $\mathcal{F}_{\phi}(r, \phi, z)=\mathcal{F}_{\phi}(r, z)$. I use a Total-Variation-Diminishing LaxFriedrich scheme (Tóth \& Odstrčil 1996) to solve the above MHD equations in conservative form. The boundary conditions are continuous everywhere except at the axis of symmetry where (a)symmetric boundary conditions were used for (momentum along the symmetry axis) density, total energy and the momentum along the $r$-axis.

\subsection{Initial conditions of the pulsar wind}

I simulate a magnetized pulsar wind by depositing mass $\dot{M}$ and energy $L$ continuously in a few grid cells concentrated around the position of the pulsar. The terminal velocity of the pulsar wind is determined from these two parameters, i.e. $v_{\infty}=\sqrt{2 L / \dot{M}_{\mathrm{pwn}}}$. In all simulations performed in this paper the terminal velocity has a value much larger then all the other velocities of interest.

I want to include the dynamical effect of the magnetic fields in the pulsar wind bubble, therefore part of the total luminosity $L_{\mathrm{tot}}$, is released as magnetic energy $L_{\mathrm{magn}}$, where the magnetization parameter $\sigma$, enters the equations:

$L_{\mathrm{magn}}=\sigma L_{\mathrm{tot}}=B^{2} / 2$.

Since we are considering a PWN which is much larger than the size $R_{\text {lc }}$ of the light-cylinder of the pulsar involved, we can safely neglect poloidal magnetic fields, therefore I will consider a purely toroidal magnetic field component. Simple estimates (cf. Contopoulos \& Kazanas 2002) give a scaling law for the ratio of the toroidal $B_{\phi}$, and poloidal $B_{\mathrm{p}}$ magnetic field strength,

$B_{\phi} / B_{\mathrm{p}} \sim R / R_{\mathrm{lc}} \gg 1$,

which makes the above assumption a valid one.

Furthermore the current calculations do not solve the problem of transforming a high $\sigma$-wind into a low $\sigma$-wind (see e.g. Lyubarsky \& Kirk 2001), the simulation already starts with a low value for $\sigma$ as is appropriate at the MHD wind termination shock (Kennel \& Coroniti 1984).

\subsection{A PWN in an uniform ISM}

The simulation of a magnetized pulsar wind interacting with a homogeneous ISM has been performed with parameters as 
Table 1: Simulation parameters

\begin{tabular}{|l|c|c|}
\hline \hline & Simulation 1 & Simulation 2 \\
\hline & & \\
Pulsar wind luminosity $L_{0}(\mathrm{erg} / \mathrm{s})$ & $1.2 \times 10^{38}$ & $1.2 \times 10^{38}$ \\
ISM mass density $\rho_{0}\left(\mathrm{~g} / \mathrm{cm}^{3}\right)$ & $1.0 \times 10^{-25}$ & $1.0 \times 10^{-25}$ \\
Terminal Velocity $v_{\infty}(\mathrm{cm} / \mathrm{sec})$ & $3.0 \times 10^{9}$ & $3.0 \times 10^{9}$ \\
Magnetization parameter $\sigma$ & $10^{-3}$ & $10^{-3}$ \\
Adiabatic Index & $4 / 3$ & $4 / 3$ \\
Explosion energy SNR $E_{0}(\mathrm{erg})$ & - & $10^{51}$ \\
Mass ejecta $M_{\mathrm{ej}}\left(M_{\odot}\right)$ & - & 3 \\
Running Time (years) & 1,100 & 1,400 \\
Number of grid cells (in r-direction) & 100 & 100 \\
Number of grid cells (in z-direction) & 320 & 320 \\
Grid size (pc) (in r-direction) & 2.5 & 2.5 \\
Grid size (pc) (in z-direction) & 8.0 & 8.0 \\
\multicolumn{2}{|l}{} & \\
\hline
\end{tabular}

denoted in Table 1 (simulation 1). The MHD code evolves till a corresponding age of $t=1100$ years. Below I will discuss the profiles of the different flow variables of the system at the end of the simulation.

Figure 1 shows the total pressure (= thermal pressure plus magnetic pressure) profile. One can observe the presence of a pressure gradient along the $r$-axis, which balances the magnetic pinching force as discussed in the previous section.

Figure 2 shows the ratio of the magnetic pressure and the thermal pressure where a cut has been taken along the $r$-axis at different positions on the $z$-axis. The model from B\&L gives a ratio of magnetic to gas pressure which is monotonically increasing as a function of the cylindrical radius $r$ and is independent of the $z$ coordinate. The model presented here allows for local maxima, which is also reflected in the magnetic field distribution shown in Fig. 3.

The density profile in Fig. 4 clearly shows the elongation of the PWN, where the ratio between the minimum and maximum distance between the shock bounding the PWN and the position of the pulsar as a function of time (denoted by $R^{-}$and $R^{+}$respectively), equals $\sim 1.5$. This results confirm the elongation of a PWN by the dynamical influence of toroidal magnetic fields inside the pulsar wind bubble as predicted by the steady-state model of B\&L.

Figure 5 shows the evolution of the minimum and maximum distance between the shock bounding the PWN and the position of the pulsar as a function of time $\left(R^{-}\right.$and $\left.R^{+}\right)$. It is shown that the expansion rate for these shock positions still follow a simple power-law expansion rate, i.e. $R^{+/-} \propto t^{\alpha}$ with $\alpha=3 / 5$ as is appropriate for the expansion law of a purely hydrodynamical (stellar) wind interacting with a uniform ISM. This indicates that although the magnetic field influences the dynamics of the PWN, the evolution of a PWN driven by a magnetized pulsar wind can still be modeled by a standard power-law expansion rate.

Figure 6 denotes the ratio between $R^{+}$and $R^{-}$, from which one can observe that a significant part of the elongation of PWNe takes place in early stages $(\sim 100$ years $)$ of its evolution. At later stages the elongation saturates at a very slow rate.

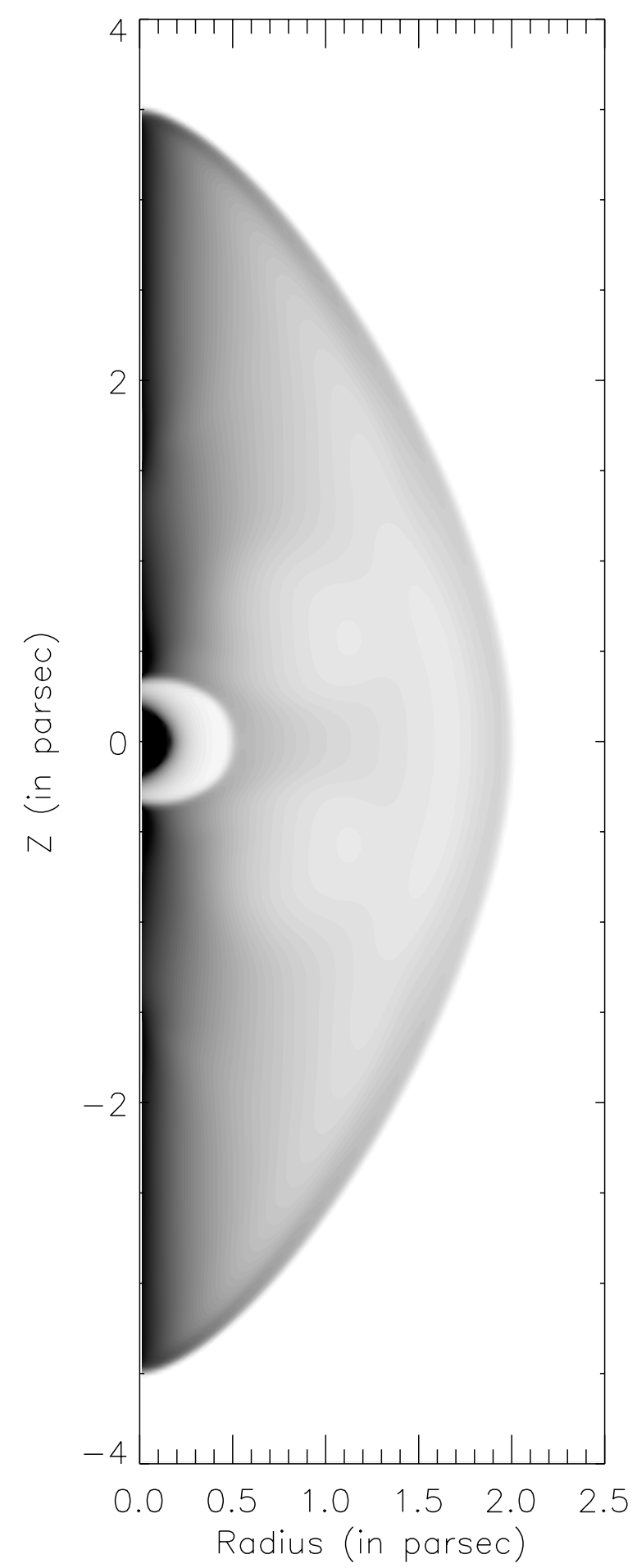

Fig. 1. Gray-scale representation of the total pressure distribution of an elongated PWN.

In the last subsection I will investigate the dependency of the elongation on the magnetization parameter $\sigma$.

\subsection{A PWN in freely expanding ejecta}

In order to investigate the structures observed in the simulations presented above are dependent on the environment in which the PWN expands, I performed a MHD simulation 

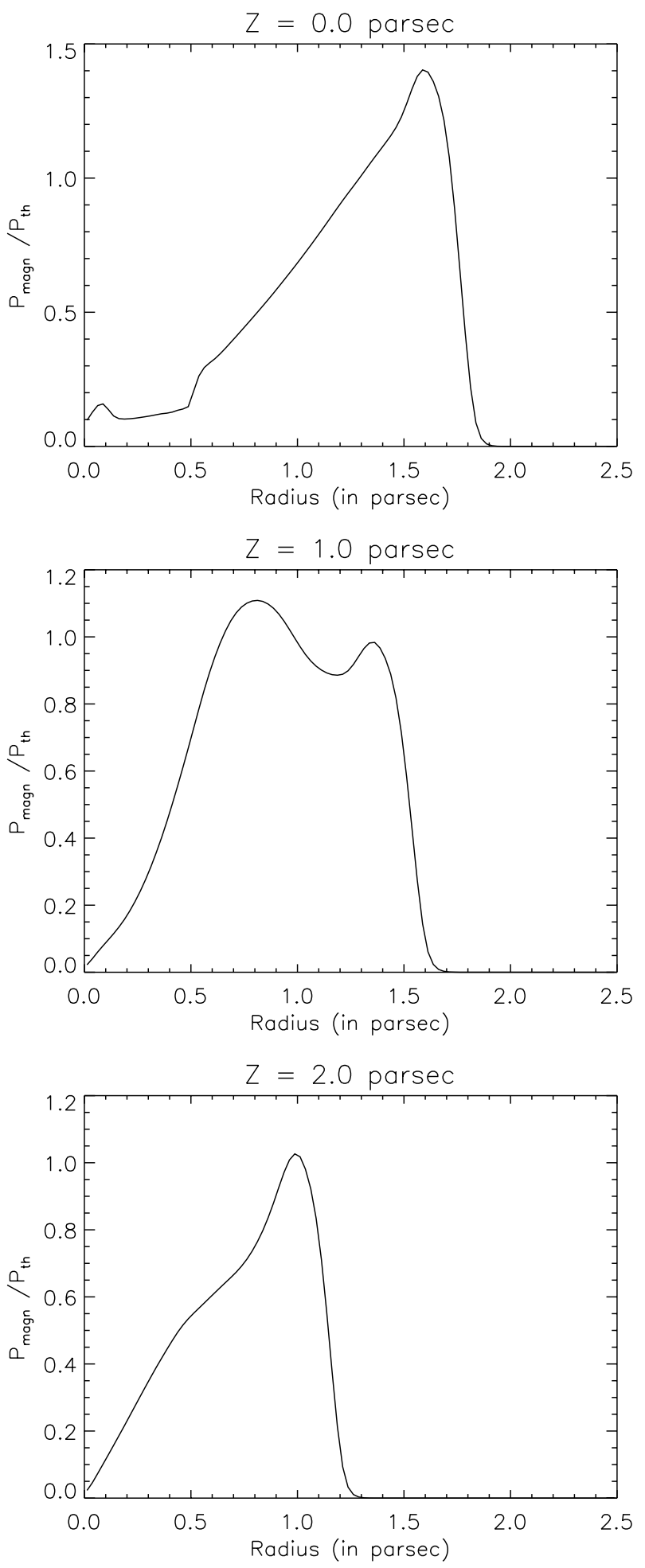

Fig. 2. Ratio of magnetic pressure $P_{\text {magn }}$, to the thermal pressure $P_{\text {th }}$ throughout the PWN. A cut has been taken along the $r$-axis for different values of $Z$.

where amagnetized pulsar wind interacts with the freely expanding ejecta of a supernova remnant. The parameters of the simulation are denoted in Table 1 (simulation 2). The freely expanding ejecta are initialised by impulsively releasing the mechanical energy and the ejected mass corresponding to a supernova explosion in the first few grid cells located around the

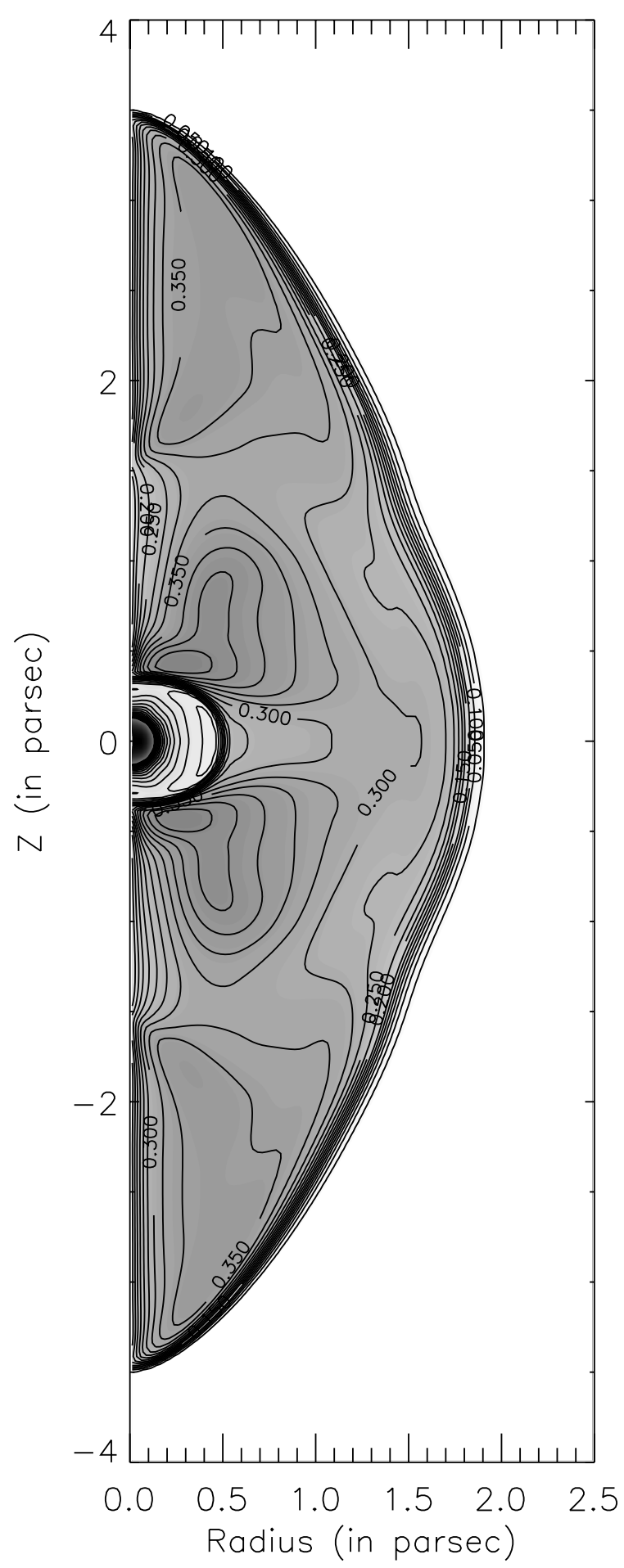

Fig. 3. Gray-scale representation of the toroidal magnetic field distribution, where the labels in the plot are in units of $10^{-4}$ Gauss.

position of the pulsar. Due to the larger scales of the expanding SNR with respect to the PWN, both the reverse shock and the forward shock will move off the grid. The freely expanding ejecta however will be represented correctly by using continuous boundary conditions, which means that the gradient is kept zero by copying the values of the flow variables from the edge of the grid into the ghost cells located around the grid. Using the above initialisation of the freely expanding ejecta of 


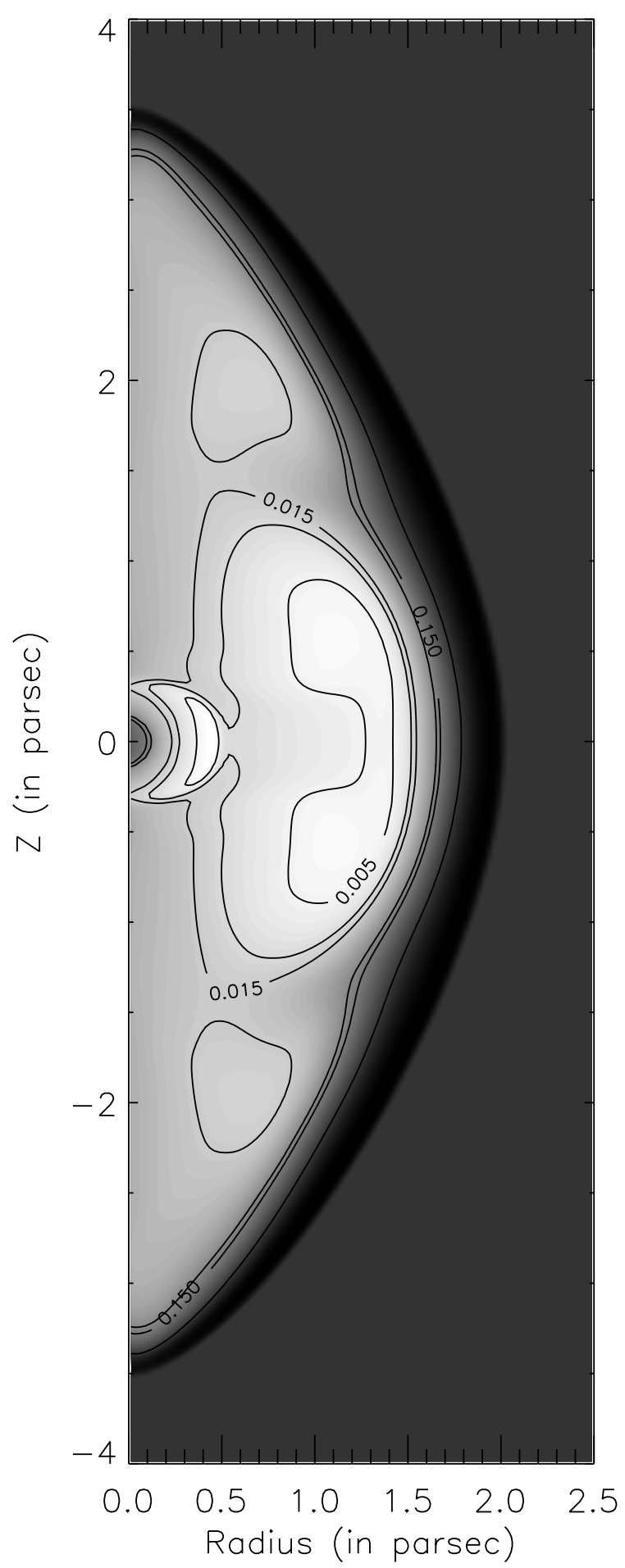

Fig. 4. Logarithmic gray-scale representation of the density distribution, where the labels in the plot are in units of $10^{-25} \mathrm{~g} / \mathrm{cm}^{3}$.

the SNR plus simultaneously simulating a pulsar wind as described above one can investigate the dynamics of the same pulsar wind as performed in simulation 1, but interacting with a different environment.

At the end of the simulation, at a corresponding age of 1400 years, the PWN has again been elongated along the symmetry axis. The overall structure of the different flow variables are similar to the ones discussed in the section above when the

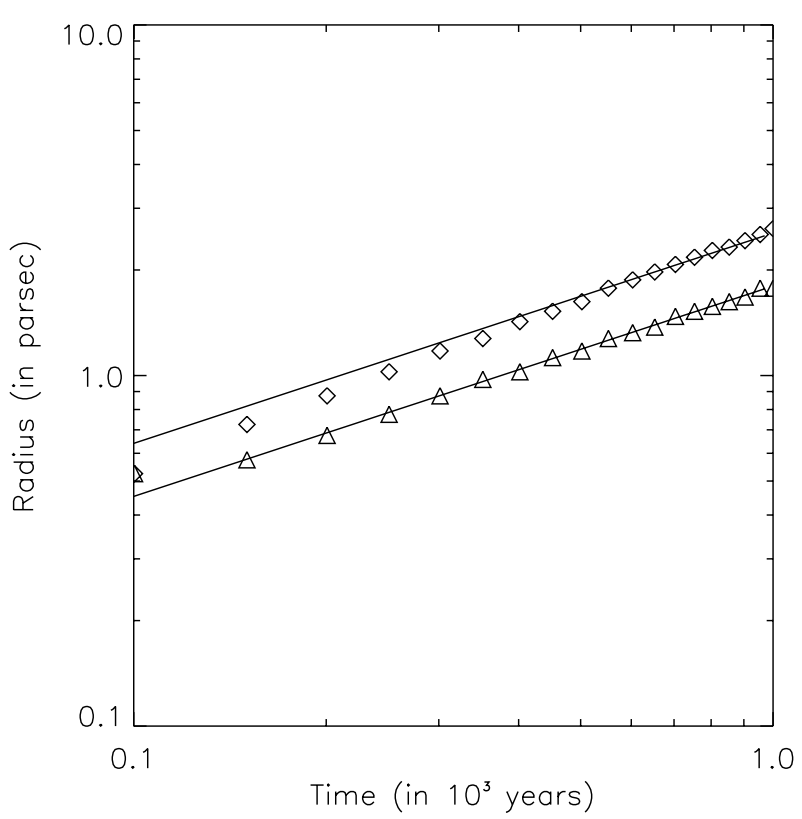

Fig. 5. Distance between the minimum (open triangles) and maximum (open squares) distance between the shock and the position of the pulsar (denoted in the text by $R^{-}$and $R^{+}$respectively). The solid lines are curves which follow a $R_{\mathrm{pwn}} \propto t^{3 / 5}$ law.

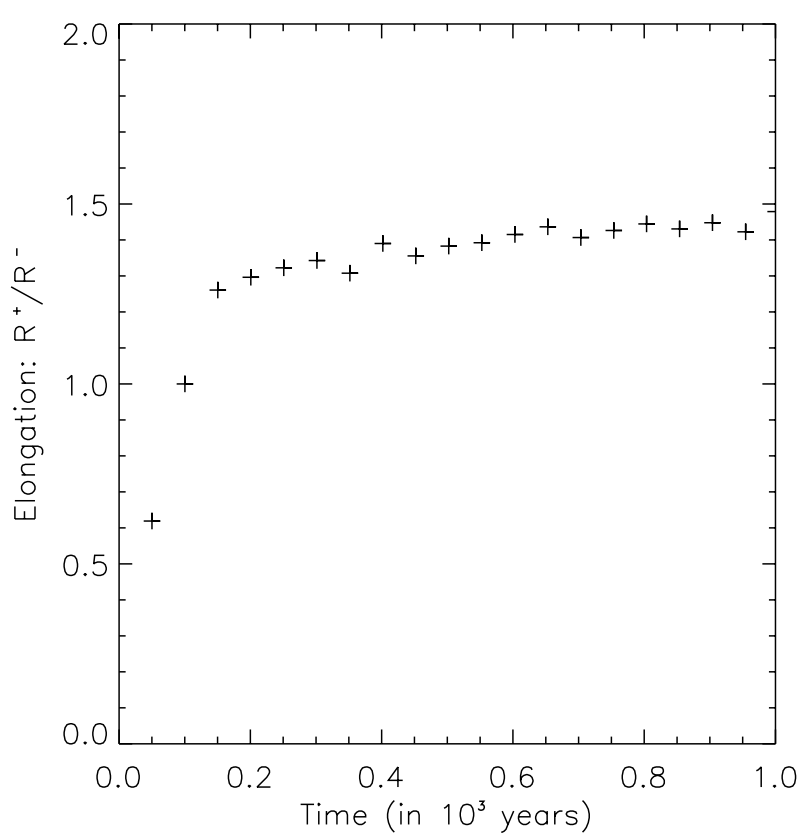

Fig. 6. Ratio of $R^{+}$to $R^{-}$, i.e. the elongation of the pulsar wind nebula due to the presence of toroidal magnetic fields.

pulsar wind was interacting with a uniform ISM. As an illustration Fig. 7 depicts the gray-scale representation of the magnetic field distribution which has the same overall structure as depicted in Fig. 3 for the first simulation.

From the above results I conclude that a magnetized pulsar wind in either a uniform ISM or in freely expanding ejecta bounded by an expanding SNR, will yield an elongated PWN with a similar distribution of the several flow variables. This seems to imply that the shape and internal dynamics of a PWN is more determined by the central engine of the system then the 


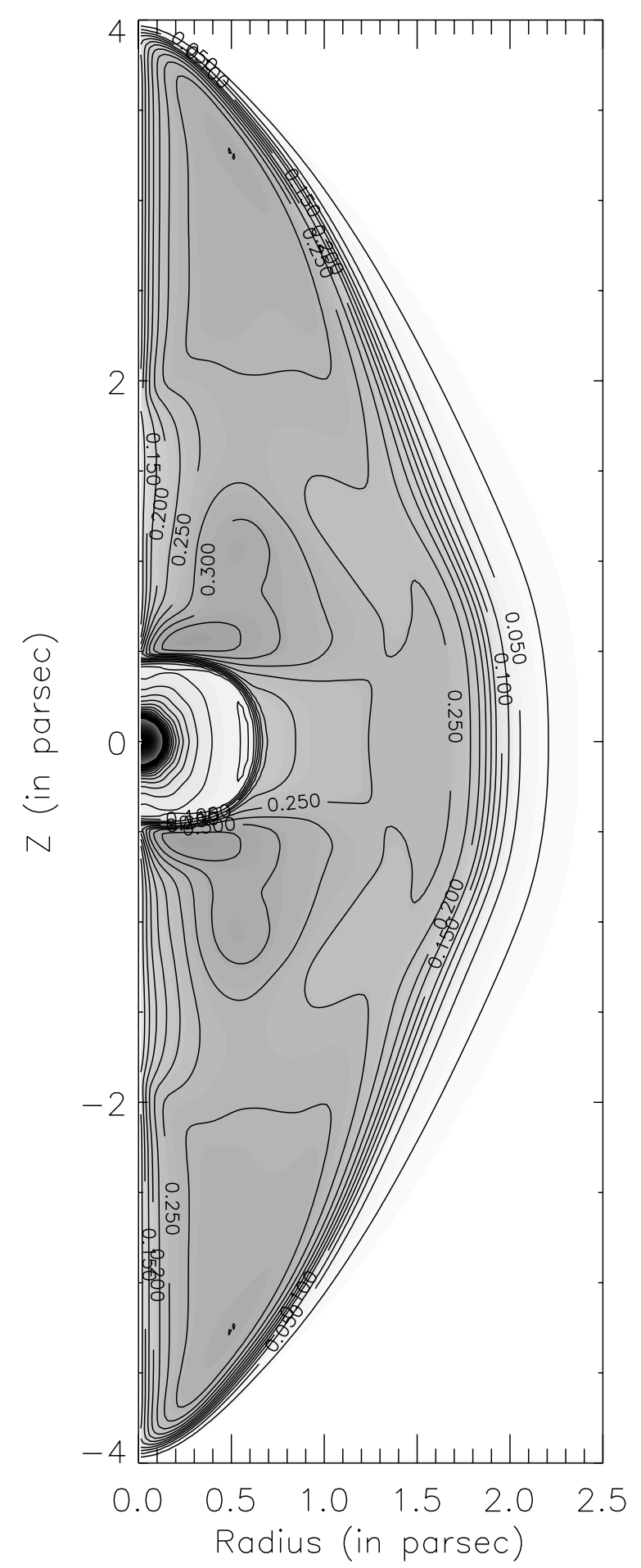

Fig. 7. Gray-scale representation of the toroidal magnetic field distribution, where the labels in the plot are in units of $10^{-4}$ Gauss.

surrounding medium in which it expands as long as the PWN is bounded by a shock.

\subsection{Elongation of pulsar wind nebulae}

The magnetization parameter determines the elongation rate of a supersonically expanding PWN. In order to quantitatively determine the influence of this parameter I performed several
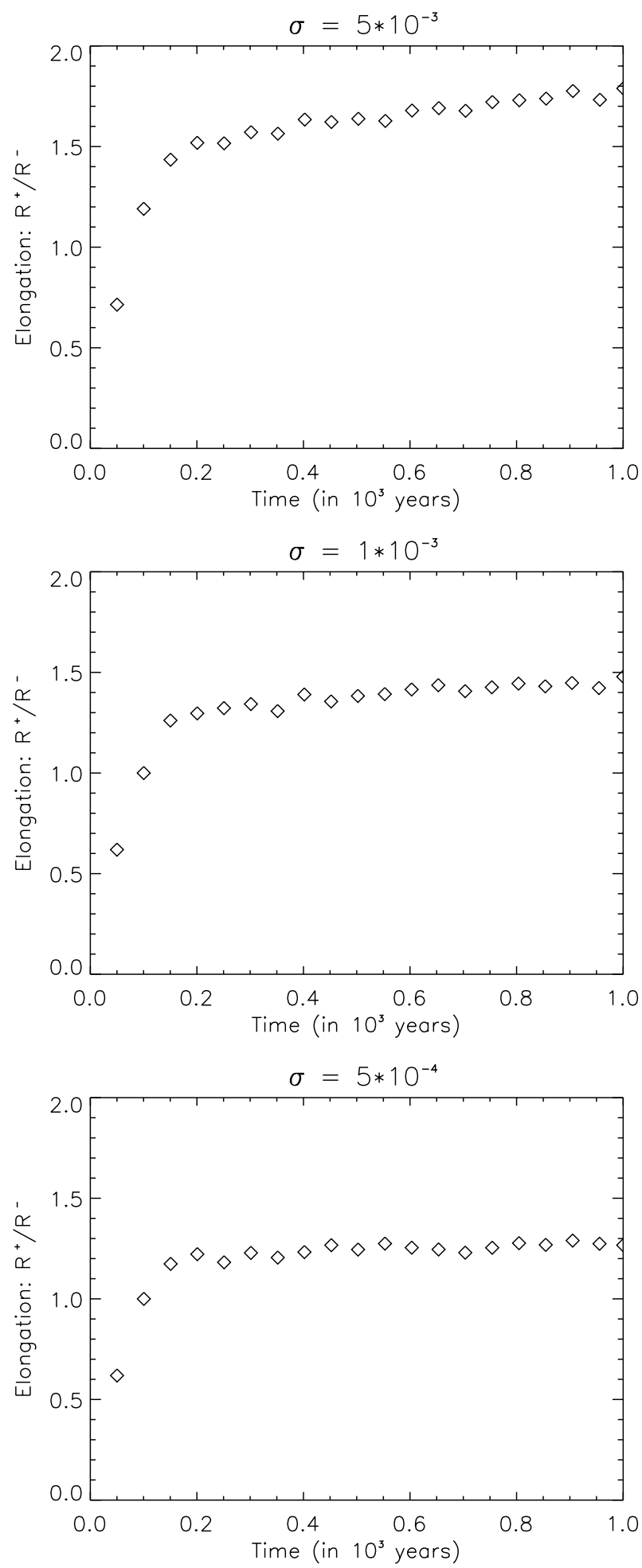

Fig. 8. Elongation of a PWN driven by the same pulsar wind (parameters are denoted in Table 1 (simulation 1)) but with different values for the magnetization parameter $\sigma$.

simulations for a magnetized pulsar wind with parameters as in Table 1 (simulation 1) but with different values for the magnetization parameter. All values are in the range of earlier models of PWNe (Kennel \& Coroniti 1984; Emmering \& Chevalier 1987; B\& L). Figure 8 summarises the results, which shows the elongation of the PWN as a function of time for the 
different MHD simulations. One can observe a weak dependency of the elongation on the magnetization parameter; an increase of roughly $\sim 1.44$ in elongation for an increase of 10 in the magnetization parameter. Furthermore, one can observe in all cases that the elongation saturates at late times in the evolution of the PWN.

\section{SNR 3C 58, a crushed PWN?}

A beautiful example of a plerionic SNR which fits the morphology of the MHD results as discussed above is $3 \mathrm{C} 58$. The shape of this remnant shows a strong elongation at radio frequencies (see for example Bietenholz et al. 2001). Recently the pulsar of this remnant has been detected, which places the active pulsar in the center of the remnant (Murray et al. 2002). This detection makes 3C 58 together with the Crab Nebula an excellent example of an elongated PWN blown into its surrounding medium by an active pulsar wind with the associated pulsar detected. I have shown in this paper that although the magnetic fields in the bubble are dynamically important and necessary to explain the elongation, the expansion of the PWN can still be modeled with a power-law expansion rate. This means that independent of the density of the surrounding medium, the expansion velocity of the PWN will roughly equal $V_{\exp }=\alpha R_{\text {pwn }} / t_{\text {age }}$, where $\alpha=6 / 5$ when expansion takes place in the freely expanding ejecta of the progenitor star and $\alpha=3 / 5$ when expansion is in an uniform ISM. Recent measurements of the radio expansion velocity of 3C 58 (Bietenholz et al. 2001) yield a value of $\sim 900 \mathrm{~km} \mathrm{~s}^{-1}$, which is in contrast with the observed values for $R_{\text {pwn }}=4.5 \mathrm{pc}$ and $t_{\text {age }}=821$ years (assuming an association with the historical SN event of 1181 and a power-law expansion rate), which would suggest an expansion velocity of $\sim 5000 \mathrm{~km} \mathrm{~s}^{-1}$.

One way out of this discrepancy is to suggest that the remnant is much older. However this would suggest that the pulsar wind is interacting directly with the ISM, and there was no freely expanding envelope due to a very low-energy supernova event (Bietenholz et al. 2001). If this scenario represents the evolutionary status of 3 C 58, the shape of 3C 58 corresponds exactly with the shape from the dynamical models presented in this paper.

Another scenario for 3C 58 was given by van der Swaluw et al. (2001), where it is suggested that the PWN of 3C 58 is being compressed by the reverse shock. This scenario can explain a number of observational properties of 3C 58 and would still associate the remnant with the presupernova progenitor of SN 1181:

- The compression of the PWN by the reverse shock would decrease the expansion velocity, which is too low in the case of 3C 58 if it was supersonically expanding (Bietenholz et al. 2001).

- The compression of the PWN increases the magnetic field and particle energies, this can lead to an increase of the radio flux density as is observed in 3C 58 in the period between 1967 and 1986 (Green 1987).

- The compression of the PWN can bring down the frequency of the synchrotron cooling break (Gallant et al. 2002), which is at a comparatively low frequency for 3C 58 (see e.g. Woltjer et al. 1997).

- The passage of the reverse shock and the subsequent compression would also increase the pressure inside the PWN. This would decrease the radius of the wind termination shock $R_{\mathrm{ts}}$. The ratio between the termination shock $R_{\mathrm{ts}}$ and the boundary of the PWN $R_{\text {pwn }}$ is indeed remarkably small in 3C 58, i.e. $R_{\mathrm{ts}} / R_{\mathrm{pwn}} \sim 0.01$ (Frail \& Moffett 1993).

If the above scenario is correct, the absence of any detected shell corresponding with the blastwave of the SNR remains a puzzle.

\section{Conclusions}

I have elaborated on the work of B\&L by performing axially symmetric 2.5D MHD simulations of a magnetized pulsar wind interacting with either a uniform interstellar medium or freely expanding ejecta. Both simulations were performed while the pulsar wind bubble is bounded by a strong shock.

The simulations only included a toroidal magnetic field component, which was injected around the position of the pulsar. The simulations show an overall decline of the total pressure in the radial direction in order to balance the magnetic pinching force due to the presence of these toroidal magnetic fields. The simulations show that the PWN is elongated as expected from the pressure differences along the PWN shock. Furthermore the simulations show that significant elongation occurs at early stages of the expansion, while at later stages the elongation saturates. Finally by performing several simulations of a pulsar wind with different values for the magnetization parameter, I conclude that the elongation depends weakly on the value of the magnetization parameter.

Although the simulations clearly show the dynamical importance of the magnetic fields, I have shown that one can still use a simple power-law expansion rate $\left(R_{\text {pwn }} \propto t^{\alpha}\right)$ to model the expansion of the PWN.

I have applied the implications of the current simulations on the naked plerion 3C 58, which is an example of a PWN driven by a pulsar wind with a detected pulsar in the center. The morphology of this plerion fits perfectly well with the simulations performed for a magnetized pulsar wind interacting with the ISM. However this would imply that the age of the remnant is much older and can not be associated with the historical supernova event in 1181. Alternatively there is observational evidence that $3 \mathrm{C} 58$ might be an example of a PWN being compressed by the reverse shock of the associated SNR. This would safeguard the association with the presupernova progenitor of SN 1181. Therefore in a future paper I will investigate the physics of a magnetized pulsar wind embedded in an expanding SNR. In such a system the reverse shock will interact with an elongated PWN and would show the evolving plerionic morphology.

Acknowledgements. I would like to thank Bram Achterberg, Luke Drury, Rony Keppens and Jan Kuijpers for useful discussions. Furthermore I would like to thank an anonymous referee for useful 
suggestions. The Versatile Advection Code was developed as part of the Massief Parallel Rekenen (Massive Parallel Computing) program funded by the Dutch Organisation for Scientific Research (NWO).

\section{References}

Begelman, M. C., \& Li, Z. Y. 1992, ApJ, 397, 187 (B\&L) Bietenholz, M. F., Kassim, N. E., \& Weiler, K. W. 2001, ApJ, 560, 772 Blondin, J. M., Chevalier, R. A., \& Frierson, D. M. 2001, ApJ, 563, 806

Castor, J. I., McCray, R., \& Weaver, R. 1975, ApJ, 200, L107

Chevalier, R. A. 1982, ApJ, 258, 790

Contopoulos, I., \& Kazanas, D. 2002, ApJ, 566, 336

Emmering, R. T., \& Chevalier, R. A. 1987, ApJ, 321, 334

Frail, D. A., \& Moffett, D. A. 1993, ApJ, 408, 637

Gallant, Y. A., van der Swaluw, E., Kirk, J. G. , \& Achterberg, A. 2002, in Neutron Stars in Supernova Remnants, ed. P. O. Slane, \& B. M. Gaensler, ASP Conf. Proc., 271, 99

Green, D. A. 1987, MNRAS, 225, 11

Kennel, C. F., \& Coroniti, F. V. 1984, ApJ, 283, 694
Lyubarsky, Y., \& Kirk, J. G. 2001, ApJ, 547, 437

McKee, C. F. 1974, ApJ, 188, 335

Murray, S. S., Slane, P. O., Seward, F. D., Ransom, S. M., \& Gaensler, B. M. 2002, ApJ, 568, 226

Rees, M. J., \& Gunn, J. E. 1974, MNRAS, 167, 1

Reynolds, S. P., \& Chevalier, R. A. 1984, ApJ, 278, 630

Sankrit, R., \& Hester, J. J. 1997, ApJ, 491, 796

Tam, C., Roberts, M. S. E., \& Kaspi, V. M. 2002, ApJ, 572, 202

Tóth, G. 1996, Astrophys. Lett. \& Comm. 34, 245

Tóth, G., \& Odstrčil, D. 1996, J. Comp. Phys., 128, 82

van der Swaluw, E., Achterberg, A., Gallant, Y. A., \& Tóth, G. 2001, A\&A, 380, 309

van der Swaluw, E., Achterberg, A., Gallant, Y. A., Downes, T. P., \& Keppens, R. 2003, A\&A, 397, 913

Wallace, B. J., Landecker, T. L., \& Taylor, A. R. 1997, AJ, 114, 2068

Wallace, B. J., Landecker, T. L., Taylor, A. R., \& Pineault, S. 1997, A\&A, 317, 212

Weiler, K. W., \& Panagia, N. 1978, A\&A, 70, 419

Woltjer, L., Salvati, M., Pacini, F., \& Bandiera, R. 1997, A\&A, 325, 295 